\title{
3-D COUPLED SIMULATION OF A VVER 1000 WITH PARCS/ATHLET
}

\author{
Romain Henry' ${ }^{1}$ Yann Périn ${ }^{1}$, Kiril Velkovªnd Sergei Pavlovich Nikonov² \\ ${ }^{1}$ Gesellschaft für Anlagen- und Reaktorsicherheit (GRS) gGmbH \\ Boltzmannstr. 14, 85748 Garching, Germany \\ ${ }^{2}$ National Research Nuclear University MEPhI \\ Kashira Highway, 31, Moscow, Russia, 115409Address \\ romain.henry@grs.de, yann.perin@grs.de,kiril.velkov@grs.de, niks.ki@mail.ru
}

\begin{abstract}
A new OECD/NEA benchmark entitled "Reactivity compensation with diluted boron by stepwise insertion of control rod cluster" is starting. This benchmark, based on high quality measurements performed at the NPP Rostov Unit 2, aims to validate and assess high fidelity multi-physics simulation code capabilities. The Benchmark is divided in two phases: assembly wise and pin-by-pin resolution of steady-state and transient multi-physics problems.

Multi-physics simulation requires the generation of parametrized few-group cross-sections. This task used to be done with deterministic (2-D) lattice codes, but in the past few years the Monte-Carlo code SERPENT has demonstrate its ability to generate accurate few-group homogenized cross-section without approximations, neither on the geometry nor in the nuclear data.

Since the whole core SERPENT models for production of such cross-section libraries would be computationally costly (and the standard 2-D approach may introduce unnecessary large approximations), 3-D models of each assembly type in infinite radial lattice configurations have been created.

These cross-sections are then used to evaluate effective multiplication factors for different core configurations with the diffusion code PARCS. The results are compared with the reference SERPENT calculations. In the next step, a thermal-hydraulic model with the system code ATHLET applying an assembly-wise description of the core (i.e. one channel per fuel assembly) has been developed for coupled PARCS/ATHLET transient test calculations.

This paper describes in detail the models and techniques used for the generation of the fewgroup parameterized cross section libraries, the PARCS model and the ATHLET model. Additionally, a simple exercise with coupled code system PARCS/ATHLET is presented and analysed.
\end{abstract}

KEYWORDS: VVER, OECD/NEA Benchmark, Monte-Carlo, ATHLET, PARCS 


\section{INTRODUCTION}

Within the framework of the Expert Group on Multi-physics Experimental Data, Benchmarks and Validation (EGMPEBV), the OECD/NEA organise a new benchmark entitled "Reactivity compensation with diluted boron by stepwise insertion of control rod cluster" [1]. Participants are invited to reproduce the transient scenario performed at the NPP Rostov Unit 2 (R2). In order to assess their results, high quality measurements are provided to the participants.

The test was performed at $75 \%$ of the nominal power $(\sim 2 \mathrm{GW})$. While the mass flow rate of the blow-down system was kept constant, the make-up system was switch off resulting in a decrease of the boron concentration of the system. The boron neutron capture being less important, the control rod cluster number 10 (Figure 1) was inserted step by step to maintain the power constant.

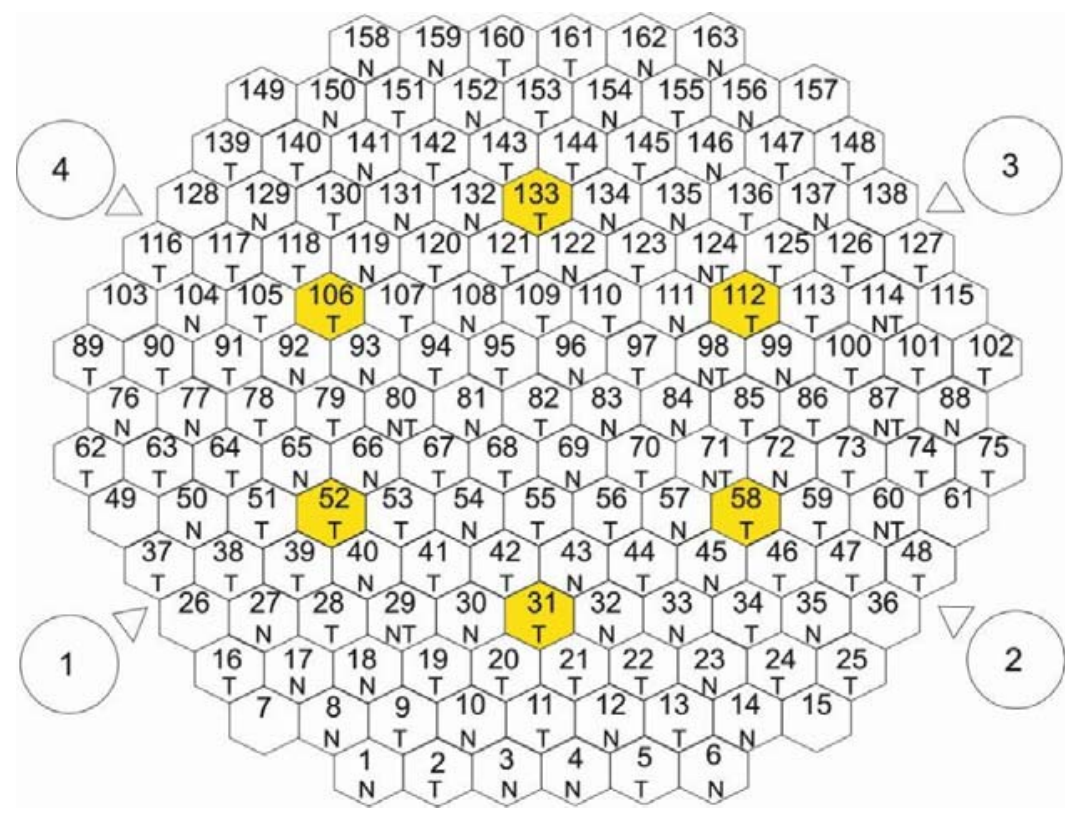

Figure 1: Core layout and position of the control rod cluster 10 (yellow)

This Benchmark aims to assess high fidelity coupled multi-physics simulation code capabilities. The Benchmark has two phases: the first one is an assembly wise resolution of the problem while the second one has a pin-by-pin description of the core and intend to reveal local 3D phenomena. The following work concerns only the assembly wise description of the problem.

In the present work, a typical VVER-1000 core is studied. First a very simple ATHLET [2] model of the core is introduced. This model is defined only for the coupling proof of concept, hence its small size, allows the resolution of problems in several minutes. Then a neutronics standalone approach, enabling the verification of the deterministic model, is detailed. The first step consists in building a reference solution with the state-of-the-art Monte-Carlo code SERPENT [3]. This model also enables the possibility to generate parameterized few-group homogenized cross-section libraries used by deterministic 3-D neutron kinetic codes such as PARCS [4]. The methodology used to produce homogenized cross-section is described in the second part of the paper. In the third section, assessment of the deterministic model is done by comparison between its predictions and the ones of the reference solution; conclusions are drawn and an outlook on the future development is given. Finally, a simple boron transient is simulated, results are shown and discussed before an outlook on the future development is given. 


\section{THERMAL-HYDRAULIC MODEL}

The thermal-hydraulic computer code ATHLET (Analysis of Thermal-hydraulics of LEaks and Transients) is being developed by the Gesellschaft für Anlagen und Reaktorsicherheit (GRS) for the analysis of operational conditions, abnormal transients and all kinds of leaks and breaks in nuclear power plants. The code is continuously enhanced and improved. For this study, a development version based on the official release version 3.1a is applied.

In this paper, a simple open-core model has been developed (Figure 2). The core is represented as 163 parallel channels without exchanges between them. Instead of modelling the primary loop of the system, boundary conditions are specified at the inlet and at the outlet of the core. At the inlet, a branching Thermofluiddynamic object is defined with a fill signal specifying Pressure, mass-flow rate, water temperature and boron concentration. At the outlet, a Time Dependant Volume branching is defined.

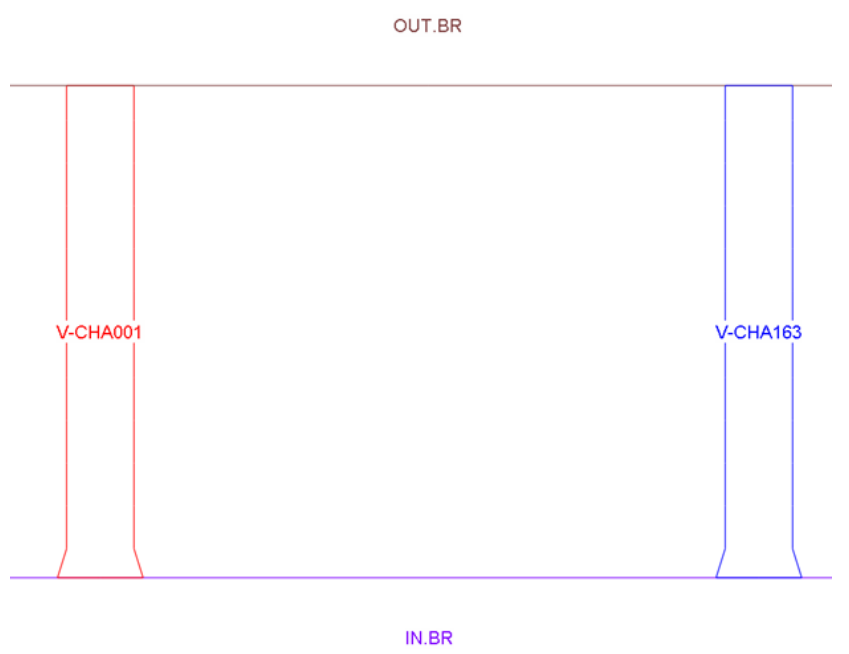

Figure 2: Scheme of the ATHLET open core model

This very simple input is only used for proof of concept in order to implement and test every model needed for the coupling between ATHLET and PARCS (control rod motion, cross-section ...).

These boundary conditions are for now fixed arbitrarily. Nevertheless, in the next step, it is foreseen to use sets of measurement data files (time dependent). In order to process the numerous data point from the Benchmark measurements, a methodology based on the plugin technology of ATHLET was created [5]. Finally, in this work the power density distribution in the reactor core is not a boundary condition but is predicted by the deterministic neutron diffusion code PARCS. The neutronic model used is described in the next section.

\section{NEUTRONIC MODEL}

\subsection{Serpent Model}

The VVER-1000 core model was built with the state-of-the-art Monte-Carlo code SERPENT and is based on the Kalinin-3 benchmark specification [6]. The core is arranged in a hexagonal lattice with a nominal pitch of $23.6 \mathrm{~cm}$ surrounded by the baffle, pierce at several position (Figure 3). Additionally, the barrel, the down comer and the reactor pressure vessel are modelled. 


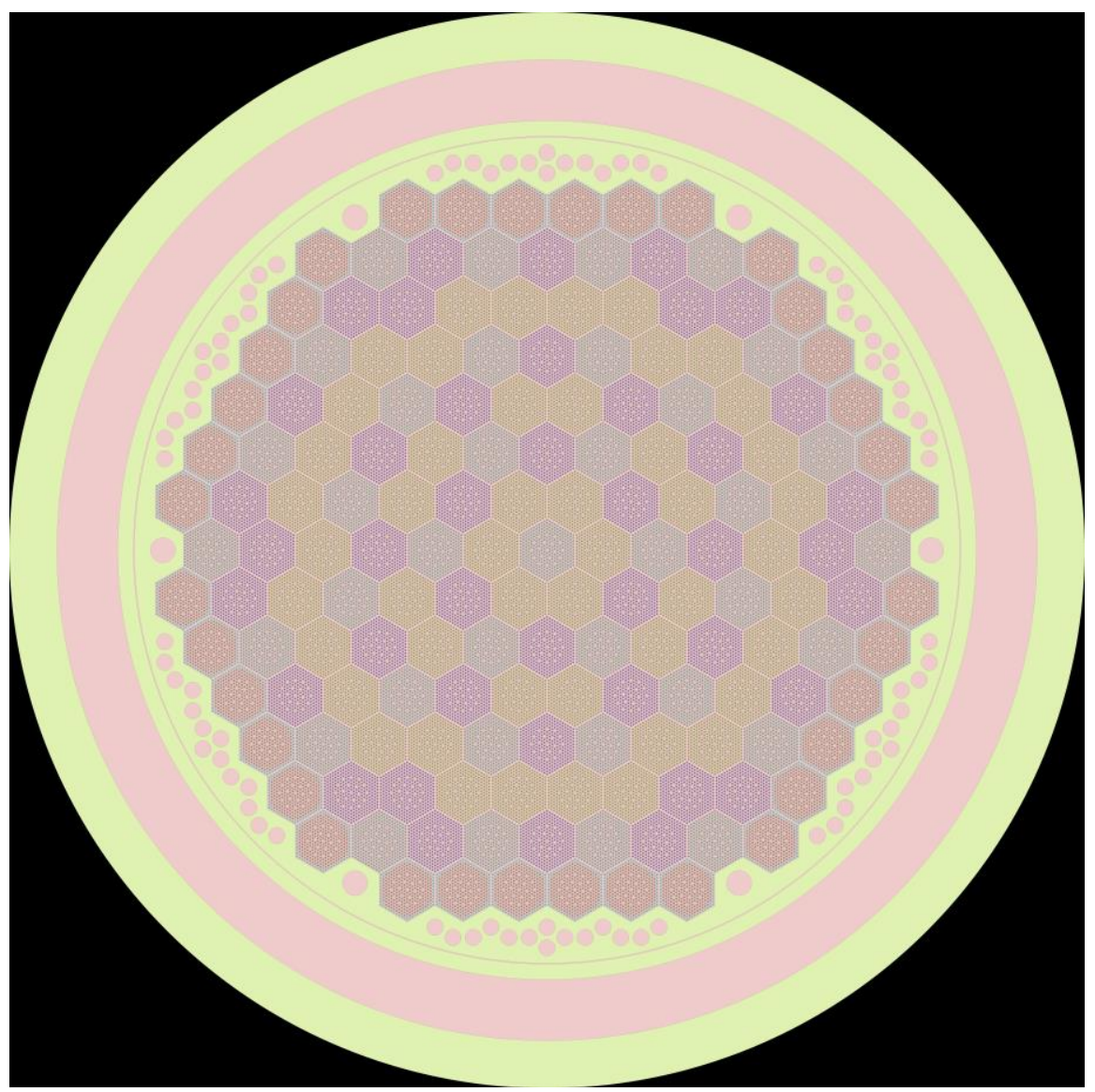

Figure 3: VVER-1000 SERPENT model, top view

The core presented here above is composed of five different fresh fuel assemblies (FA):

- 48 FA with $\mathrm{U}^{235}$-enrichment of $1.3 \%$

- 42 FA with $\mathrm{U}^{235}$-enrichment of $2.2 \%$

- $\quad 37$ FA with average $\mathrm{U}^{235}$-enrichment of $2.98 \%$ (fuel rod with gadolinium)

- 24 FA with average $U^{235}$-enrichment of $3.9 \%$ (fuel rod with gadolinium)

- 12 FA with average $\mathrm{U}^{235}$-enrichment of $3.9 \%$ (fuel rod with gadolinium)

More detailed information can be found in the benchmark definition [6].

The nuclear data library used for criticality calculations was JEFF 3.1.1. In order to ensure a standard deviation smaller than $1 \mathrm{pcm}$ for multiplication factors, 1000 cycles of $10^{7}$ neutron histories each have been simulated, first 100 batches were discarded. Results are presented in the section 4 together with deterministic predictions.

Besides providing a reference solution to our problem, SERPENT modelling allow the generation of homogenised cross-section. In the next section, the methodology applied to obtain the different group constant required to solve the neutron diffusion equation is presented. 


\subsection{Cross-Section Homogenization for Deterministic Calculation}

The first step in a deterministic calculation is to generate macroscopic cross-sections. This task used to be done by deterministic (2-D) lattice codes, but in the past few years SERPENT has shown its ability to generate accurate few-group homogenized cross-section without approximations, on the geometry. The main drawback of the Monte-Carlo method remains the large computational power required. This is why for fuel assembly, a single FA (Figure 4, left) is considered with reflective boundary conditions for the generation of cross-sections. For reflector, the simulation domain to obtain the cross-section is the full core. 10 hexagonal domains are considered (Figure 4, right). Indeed, in the vicinity of the core, the ratio between water gap and solid structure is very irregular causing non negligible difference from one hexagonal area to the other. Significant improvement has been observed here with PARCS, while using 10 reflector areas, for power distribution prediction.

To achieve the desired accuracy in cross-section generation, SERPENT calculations were performed with 1000 cycles of $2.10^{5}$ neutron histories each, first 100 batches were discarded. Different states (fuel temperature, water density and boron concentration) were modelled to produce parametrize few-group cross-section library and assembly discontinuity factors.
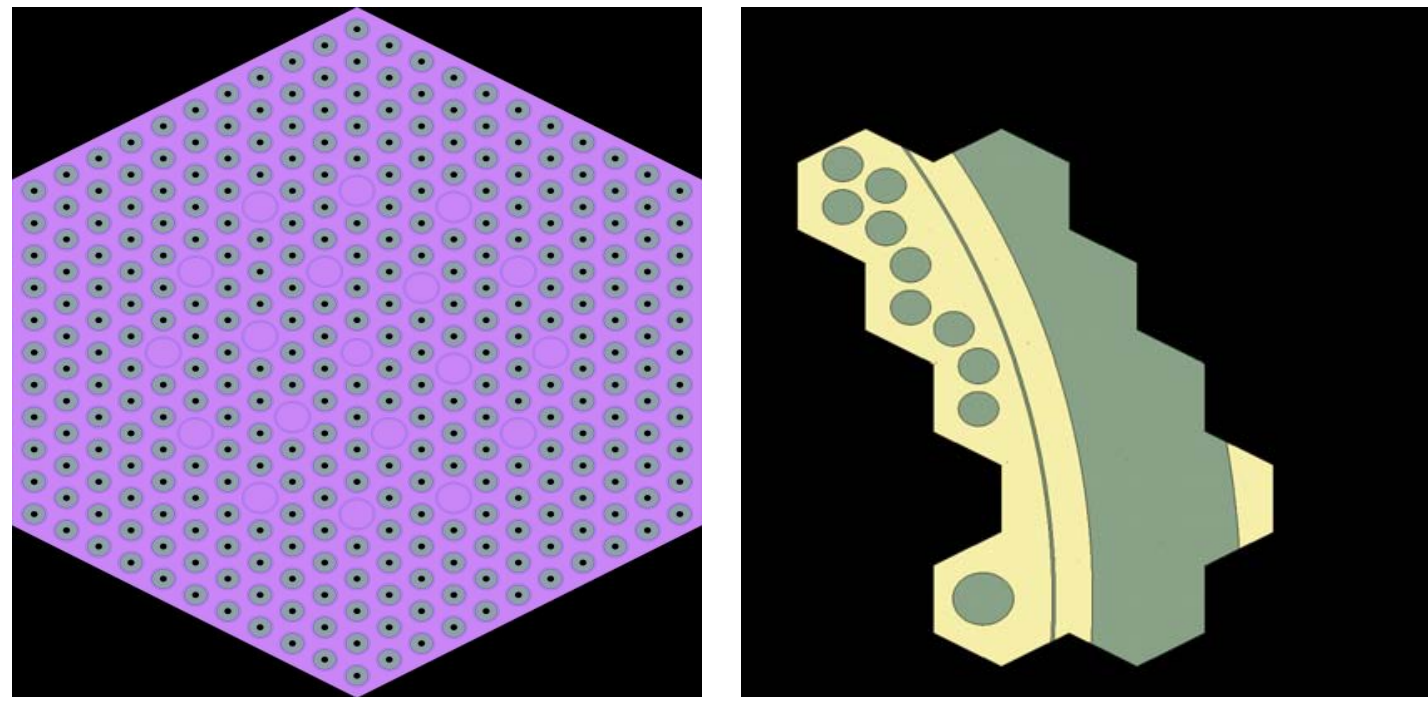

Figure 4: single FA model (left), cell reflector model (right)

\subsection{Results}

In order to assess the accuracy of PARCS model and of the Homogenise cross-section, several criticality calculations, for different state, were run. Results of SERPENT and PARCS are summarise in Table I.

First of all, very good agreement can be observed between PARCS and SERPENT with a maximum difference smaller than $100 \mathrm{pcm}$. Additionally, assembly power distributions have been compared and a maximum discrepancy of $1.8 \%$ has been observed (Figure 5 ). The ring symmetry of the power distribution deviations shows the influence of the different reflector modelling of the two codes which can be decreased in the future by applying some reflector corrections. 
Table I : Effective multiplication factor for different core configuration

\begin{tabular}{|c|c|c|c|c|c|}
\hline $\begin{array}{c}\text { Fuel } \\
\text { temperature } \\
(\mathrm{K})\end{array}$ & $\begin{array}{c}\text { Moderator } \\
\text { density } \\
(\mathrm{kg} / \mathrm{m} 3)\end{array}$ & $\begin{array}{c}\text { Boron } \\
\text { concentration } \\
(\mathrm{ppm})\end{array}$ & SERPENT (S) & PARCS (P) & $\begin{array}{c}\text { Reactivity difference: } \\
\text { S-P (pcm) }\end{array}$ \\
\hline 300 & 842.8 & 0 & 1.22518 & 1.22438 & 53 \\
\hline 300 & 842.8 & 900 & 1.06634 & 1.06535 & 87 \\
\hline 300 & 661.1 & 0 & 1.18618 & 1.18593 & 18 \\
\hline 300 & 661.1 & 900 & 1.05604 & 1.05616 & -10 \\
\hline 600 & 842.8 & 0 & 1.21375 & 1.21305 & 48 \\
\hline 600 & 842.8 & 900 & 1.05647 & 1.05554 & 84 \\
\hline 600 & 661.1 & 0 & 1.17366 & 1.17343 & 17 \\
\hline 600 & 661.1 & 900 & 1.04493 & 1.04518 & -23 \\
\hline 900 & 842.8 & 0 & 1.20507 & 1.20440 & 46 \\
\hline 900 & 842.8 & 900 & 1.04890 & 1.04798 & 84 \\
\hline 900 & 661.1 & 0 & 1.16412 & 1.16400 & 9 \\
\hline 900 & 661.1 & 900 & 1.03655 & 1.03691 & -34 \\
\hline
\end{tabular}

0.00 .00 .20 .20 .40 .1

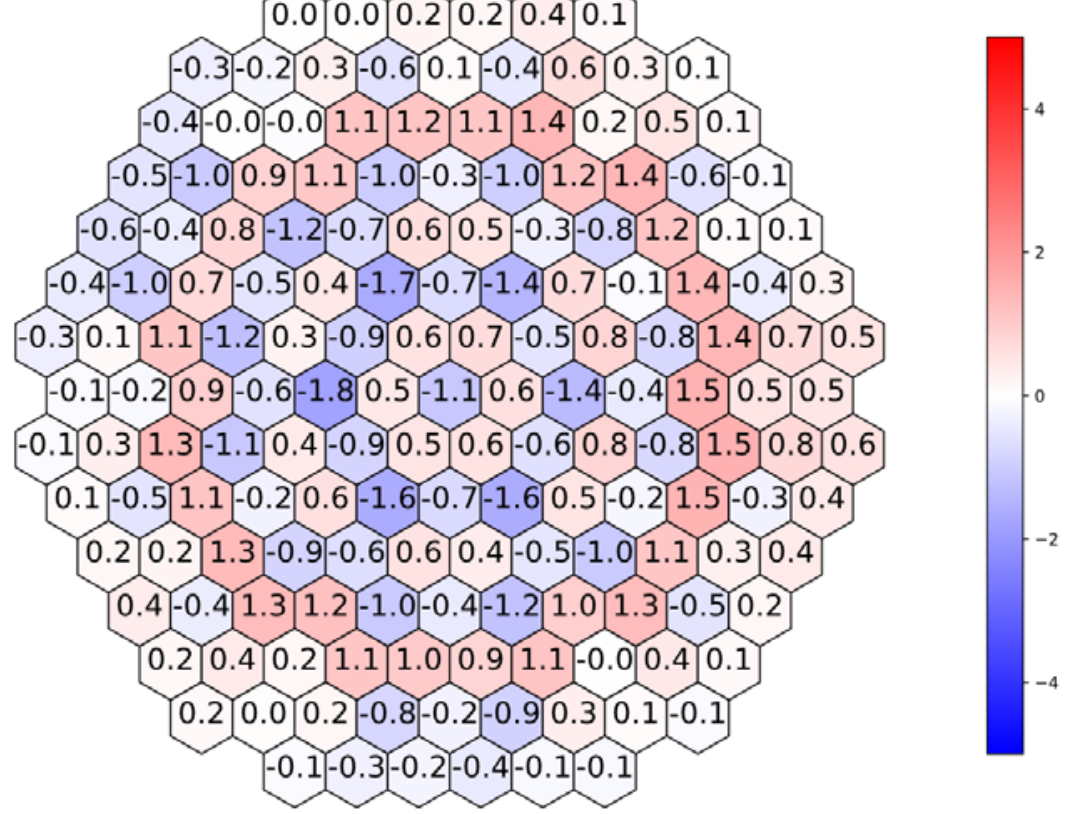

Figure 5: Relative assembly power differences between PARCS and SERPENT

\section{COUPLING SIMULATION}

The models of ATHLET and PARCS have a 1-by-1 mapping scheme, i.e. each subassembly is represented by an individual thermal-hydraulic parallel channel, the traditional GRS coupling scheme between ATHLET and a neutronics solver has been used [7]. For the first test scenario, a Rostov-2 alike boron 
transient was chosen. This choice was made to prepare, as much as possible, the Rostov-2 benchmark (data were not yet release at that time).

In this transient the critical boron concentration is continuously decreased to simulate a boron dilution (Figure 6). In order to compensate positive reactivity inserted in the core by the boron dilution, control rod cluster 10 (Figure 1) is inserted twice by $14 \mathrm{~cm}$ at a speed of $2 \mathrm{~cm} \cdot \mathrm{s}^{-1}$.

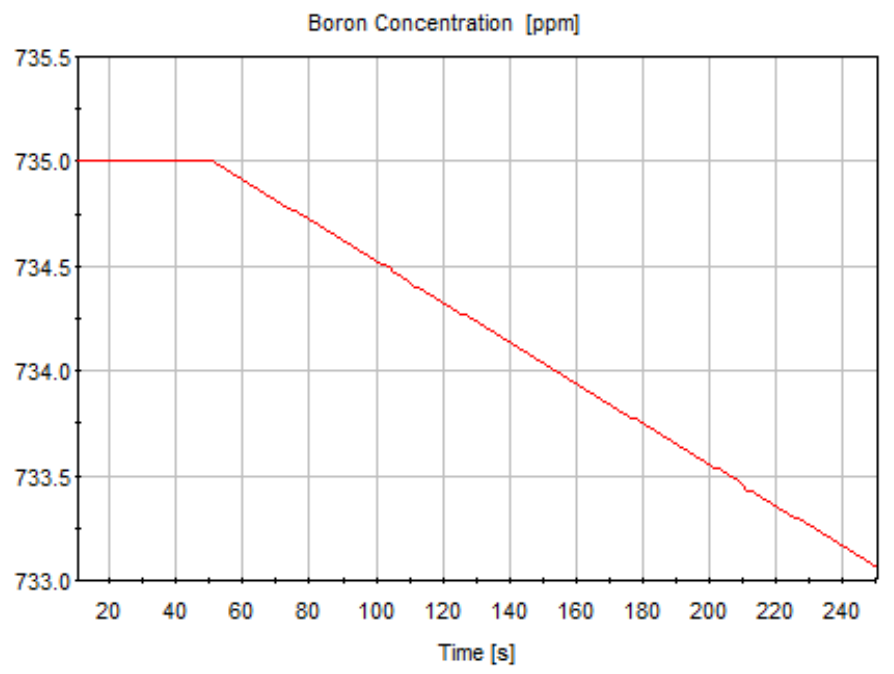

Figure 6: Time evolution of the Boron concentration

As a result, one can see (Figure 7, left) that due to a diminution of neutron absorptions by the boric acid, the power increases slowly. At every insertion of the control rod cluster, a sudden decrease of the power is observed (as expected). This is then followed by a power rise that can be decomposed in two parts. First, a sudden rise of the power is induced by the fuel Doppler effect. Hence, the sudden decrease of the power impact directly the fuel temperature (Figure 7, right) and trigger the negative thermal feedback of the fuel. The second part is simply the result of the boron dilution insertion.

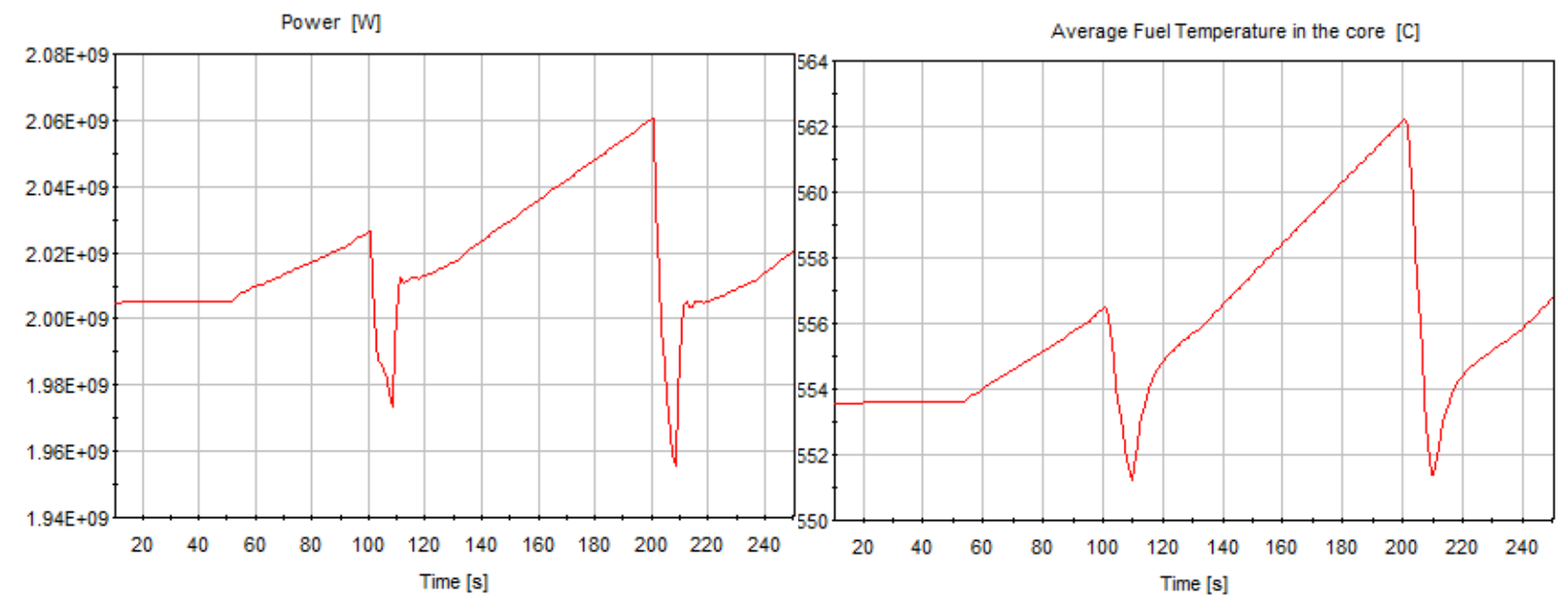

Figure 7: Time evolution of the Power (left) and of the average fuel temperature (right) 
Naturally, the 'value' of these results do not lie in the numerical predictions but in the ability of the coupled model to produce physically plausible result induced by an initial small perturbation (few ppm). Indeed, it appears that every effect is well described by the model (Boron dilution, control rod motion, fuel Doppler). However, the boron reactivity coefficient was found to be $30 \%$ higher than in reality. An investigation was carried out and concludes that the support point without boron and far away from the real boron concentration was responsible. Nevertheless, this should not happen during the resolution of the benchmark. All in all, this test exercise was successful and pave the way toward the resolution of the R-2 benchmark.

\section{CONCLUSIONS}

The OECD/NEA "Rostov-2" is an opportunity to validate and assess high fidelity multi-physics simulation code capabilities. In order to prepare the benchmark, codes traditionally used at GRS: ATHLET for thermalhydraulics and SERPENT and PARCS for the neutron physics were used to prepare input decks. First a simple open core model of VVER 1000 was built with ATHLET. This model is defined for the coupling proof of the concept; allow the resolution of problems in several minutes. Additionally, in order to perform coupled multi-physics calculations, a full core VVER-1000 core was built with SERPENT. This model is a reference solution but also allow the generation of few-group cross-sections for the deterministic code PARCS. Preliminary results have shown good agreement between PARCS and reference SERPENT solutions. Finally, in order to test the coupled model, a Rostov-2 alike boron transient was resolved. The corresponding transient was simulated and has shown results physically plausible Every effect was well described by the coupled model (Boron dilution, control rod motion, fuel Doppler). In the future, real data from the benchmark are foreseen to be used to perform the first phases of the benchmark and move toward pin by pin resolution of the problem.

\section{REFERENCES}

[1] M. Avramova, K.Ivanov, K.Velkov, I. Pasichnyk, S.P.Nikonov, M. Denisova, A. Denisenko, P. Gordienko, B. Shumskiy, Benchmark on reactivity compensation with diluted boron by stepwise insertion of control rod cluster into the VVER-1000 core, Specification and Support Data v. 1.3 (2018).

[2] G. Lerchl, H. Austregesilo, P. Schöffel, D. von der Cron, F. Weyermann, ATHLET Mod3.1 Cycle A, User's Manual (2016).

[3] J. Leppänen, M. Pusa, T. Viitanen, V. Valtavirta, T. Kaltiaisenaho, The Serpent Monte Carlo code: Status, development and applications in 2013, Annals of Nuclear Energy 82 (2015) $142-150$.

[4] T.J. Downar, D.A. Barber, R.M. R. Miller, C. Lee, T. Kozlowski, D. Lee, Y. Xu, J. Gan, H.G. Joo, J.Y. Cho, K. Lee, A.P. Ulses, PARCS: Purdue Advanced Reactor Core Simulator PHYSOR 2002, Seoul, Korea, October 7-10, 2002 (2012).

[5] Y. Perin, R. Henry, K. Velkov, S. Nikonov, Transient analysis of a detailed thermal-hydraulic model of a VVER-1000 core with the system code ATHLET, in: NURETH 18th USA, Portland 2019.

[6] V. A. Tereshonok, V. S. Stepanov, V. V. Ivchenkov, V. A. Pitilimov, S. P. Nikonov, Description of a Transient Caused by the Switching-off of One of the Four Operating MCP at Nominal Reactor Power at NPP Kalinin Unit 3 (2008).

[7] S.Langenbuch and K. Velkov, Overview on the Development and Application of the Coupled Code System ATHLET-QUABOX/CUBBOX, in: Proceedings of M\&C 2005 - Mathematics and Computation, Supercomputing, Reactor Physics and Nuclear and Biological Applications, 2005. 